\title{
Peritumoral lymphatic invasion is associated with regional lymph node metastases in prostate adenocarcinoma
}

\author{
Andres A Roma ${ }^{1}$, Cristina Magi-Galluzzi ${ }^{1}$, Melinda A Kral ${ }^{1}$, Tao T Jin ${ }^{2}$, Eric A Klein ${ }^{3}$ and \\ Ming Zhou ${ }^{1}$ \\ ${ }^{1}$ Department of Anatomic Pathology, Cleveland Clinic Foundation, Cleveland, OH, USA; ${ }^{2}$ Department of \\ Quantitative Health Sciences, Cleveland Clinic Foundation, Cleveland, OH, USA and ${ }^{3}$ Glickman Urological \\ Institute, Cleveland Clinic Foundation, Cleveland, OH, USA
}

\begin{abstract}
Lymphangiogenesis, detected by antibodies specific for lymphatic endothelial cells, has been associated with regional lymph node metastases and poor prognosis in carcinomas of head and neck, breast and uterine cervix, but remains largely uninvestigated in prostate adenocarcinoma. We evaluated the lymphatic vessel density and lymphatic vessel invasion by prostate cancer cells in the intratumoral, peritumoral and normal prostate tissue compartments in cancer-bearing prostate glands and correlated them with lymph node metastases, Gleason score and other pathological parameters. Lymphatic vessels were detected by immunohistochemical stain using an antibody specific for the lymphatic endothelial cells (clone D2-40) on 33 radical prostatectomies. In all, 26 patients had lymph node dissection, and 14 of them had lymph node metastasis. The lymphatic vessel density and lymphatic vessel invasion were then recorded for each of the three compartments microscopically. Lymphatic vessel density in the intratumoral, peritumoral and normal prostate compartments was $0.91 \pm 0.80$, $1.54 \pm 0.68$ and $1.58 \pm 0.96 / \mathrm{mm}^{2}$, respectively. The intratumoral lymphatic vessel density was significantly lower than that of the peritumoral and normal prostate compartments, and the latter two were not significantly different. The lymphatic vessel density of the three compartments was not significantly different between cases with and without lymph node metastasis. The peritumoral lymphatic vessel density correlated inversely with the Gleason score. Lymphatic vessel invasion was present in significantly higher percentage of cases with lymph node metastasis $(9 / 14,62.3 \%)$, as compared to those without lymph node metastasis $(1 / 12,8.3 \%, P<0.01)$. The peritumoral lymphatic vessel invasion had a better correlation with the presence of lymph node metastases than intratumoral lymphatic vessel invasion. There is no evidence of lymphangiogenesis in prostate adenocarcinoma. Peritumoral lymphatic vessel invasion correlates with regional lymph node metastases, suggesting that the peritumoral lymphatic vessels are functionally important and identification of lymphatic vessel invasion in this compartment implies a high probability of regional lymph node metastases. Modern Pathology (2006) 19, 392-398. doi:10.1038/modpathol.3800546; published online 6 January 2006
\end{abstract}

Keywords: prostate adenocarcinoma; lymphatic vessels; lymphangiogenesis; lymph node metastases

Metastasis is the hallmark of malignant neoplasms and the lymphatic system provides a key route for neoplastic dissemination. ${ }^{1,2}$ Recent experimental evidence suggests that a wide variety of tumor cells can produce several lymphangiogenic factors, including vascular endothelial growth factor $\mathrm{C}$ and $\mathrm{D}$ (VEGF C and D), that promote the growth and remodeling of lymphatic vessels, a process termed lymphangiogenesis, through interaction with a cell

Correspondence: Dr M Zhou, MD, PhD, Department of Anatomic Pathology, Cleveland Clinic Foundation, 9500 Euclid Avenue/ L25, Cleveland, OH 44195, USA.

E-mail: zhoum@ccf.org

Received 10 November 2005; revised 22 November 2005; accepted 23 November 2005; published online 6 January 2006 surface receptor tyrosine kinase, vascular endothelial growth factor receptor 3 (VEGFR-3). ${ }^{2-6}$ Studies have also demonstrated that the expression of these lymphangiogenic factors correlate with metastatic spread of tumor cells to lymph nodes in many solid tumors. ${ }^{2-6}$ Furthermore, lymphangiogenesis detected by antibodies specific for lymphatic endothelial cells, has also been associated with regional lymph node metastases and poor prognosis in head and neck squamous cell carcinoma, melanoma, cervical and pancreatic cancers, ${ }^{7-11}$ although recent studies on breast cancer seem to contradict this finding. ${ }^{12,13}$

Prostate adenocarcinoma is the most common noncutaneous malignancy and the second-leading cause of cancer death among men in the United 
States. ${ }^{14,15}$ The presence of lymph node metastasis is a poor prognostic sign and indicates that the therapeutic modalities of curative intent, such as radical prostatectomy and radiation, have low probability of success in these patients. ${ }^{16}$ The molecular mechanism underlying lymphatic invasion and regional lymph node metastases are poorly understood in prostate cancer. Several studies have found an increased expression of lymphangiogenic growth factors VEGF-C and D, and their receptor VEGFR-3 in prostate cancer cells and the increased expression of these genes correlated with advanced disease stage and lymph node metastases. ${ }^{17-20}$ However, little is known whether the increased expression of these lymphangiogenic factors, in fact, leads to quantitative and/or qualitative alteration of lymphatic vessels in prostate adenocarcinoma or adjacent prostate tissue in prostate adenocarcinomabearing prostate glands. Only one recent study demonstrated lack of lymphangiogenesis in prostate adenocarcinoma. ${ }^{21}$ The same study also showed no correlation between the lymphatic vessel density and other pathological parameters. ${ }^{21}$

The objectives of our study were to confirm the lack of lymphangiogenesis in prostate adenocarcinoma by comparing the lymphatic vessel density in intratumoral, peritumoral and normal prostate compartments in prostate glands harboring prostate adenocarcinoma; to assess whether lymphatic vessels are functional, that is, whether invasion of these lymphatic vessels leads to lymph node metastasis; and to correlate lymphatic vessel density and lymphatic vessel invasion with other pathological parameters, including lymph node status, Gleason score, tumor volume, extraprostatic extension, seminal vesicle invasion and surgical margin status.

\section{Materials and methods}

\section{Case Selection}

A total of 33 radical prostatectomy specimens performed for prostate adenocarcinoma at the authors' institution between 1998 and 2004 were included in this study. The clinical and pathological parameters of these cases were summarized in Table 1. In all, 26 patients had pelvic lymphadenectomy, 14 of whom had metastases in the pelvic lymph nodes involving a mean of 1.5 lymph nodes (range 1-3). Seven patients had no lymph node dissection. A single paraffin block containing prostate adenocarcinoma representative of the entire case (same Gleason score as the overall score of the case) was chosen for immunohistochemical detection of lymphatic vessels.

\section{Detection of Lymphatic Vessels by Immunohistochemistry}

Immunohistochemistry for lymphatic vessels was performed using a mouse monoclonal antibody
Table 1 Clinical and pathological parameters of 33 prostate adenocarcinomas

\begin{tabular}{lr}
\hline Age: mean (range) & $60.5(46-70)$ \\
Gleason score $^{\mathrm{a}}$ & \\
$\quad 6$ & $3 / 33(9.1 \%)$ \\
7 & $23 / 33(69.7 \%)$ \\
$\geq 8$ & $7 / 33(21.2 \%)$ \\
Tumor volume & \\
$\quad$ Low & \\
Medium & $1 / 33(3 \%)$ \\
Extensive & $10 / 33(30.3 \%)$ \\
Extraprostatic extension & $22(66.7 \%)$ \\
Seminal vesicle invasion & $18 / 33(54.5 \%)$ \\
Positive surgical margin & $12 / 33(36.4 \%)$ \\
Lymph node metastasis & $11 / 33(33.3 \%)$ \\
\end{tabular}

${ }^{\mathrm{a}}$ Two patients had preoperative androgen ablation therapy and the Gleason scores in preoperative needle biopsies were used for the final grades.

${ }^{\mathrm{b}}$ The tumor volume was calculated based on the method by Renshaw et $a{ }^{29}$ Low tumor volume $<0.5 \mathrm{ml}^{3}$, medium tumor volume $0.5-$ $2 \mathrm{ml}^{3}$, and large tumor volume $>2 \mathrm{ml}^{3}$.

reactive with an O-linked sialoglycoprotein found on lymphatic endothelium (clone D2-40, Signet Laboratories, Dedham, MA, USA) ${ }^{8,22,23}$ Briefly, the $5-\mu \mathrm{m}$ tissue sections were antigen-retrieved in $0.1 \mathrm{M}$ citrate buffer, pH6.0, in a pressure steamer for $15 \mathrm{~min}$. The slides were then incubated sequentially with primary antibody (1:2000 dilution), biotinylated secondary antibody, avidin-peroxidase complex (Ventana, Tucson, AZ, USA) and chromogenic substrate diaminobenzidine.

\section{Quantification of Lymphatic Vessels and Lymphovascular Invasion}

The three compartments of the prostate gland were defined as followings. The intratumoral compartment was defined as the area encompassing all the cancer glands present in the representative H\&E section. The peritumoral compartment was a $1 \mathrm{~mm}-$ wide area around the intratumoral compartment. The area beyond the peritumoral compartment was defined as normal prostate compartment. The three compartments were marked on the $\mathrm{H} \& \mathrm{E}$ slides and their areas $\left(\mathrm{mm}^{2}\right)$ were calculated using a $1 \mathrm{~mm}$ grid. The numbers of lymphatic vessels in these three zones were counted under the microscope using $10 \times$ objectives. Any discrete D2-40 positive structure, regardless of the presence of lumens, was counted as one lymphatic vessel. Although basal cells of the benign prostate glands were also positive for D2-40, they could easily to be distinguished from lymphatic vessels (Figure 1). Lymphatic vessel density, defined as the number of lymphatic vessels per compartmental area, was calculated. Lymphatic vessel invasion, defined as prostate adenocarcinoma cells within the D2-40 positive structures, was also recorded for the three compartments. 

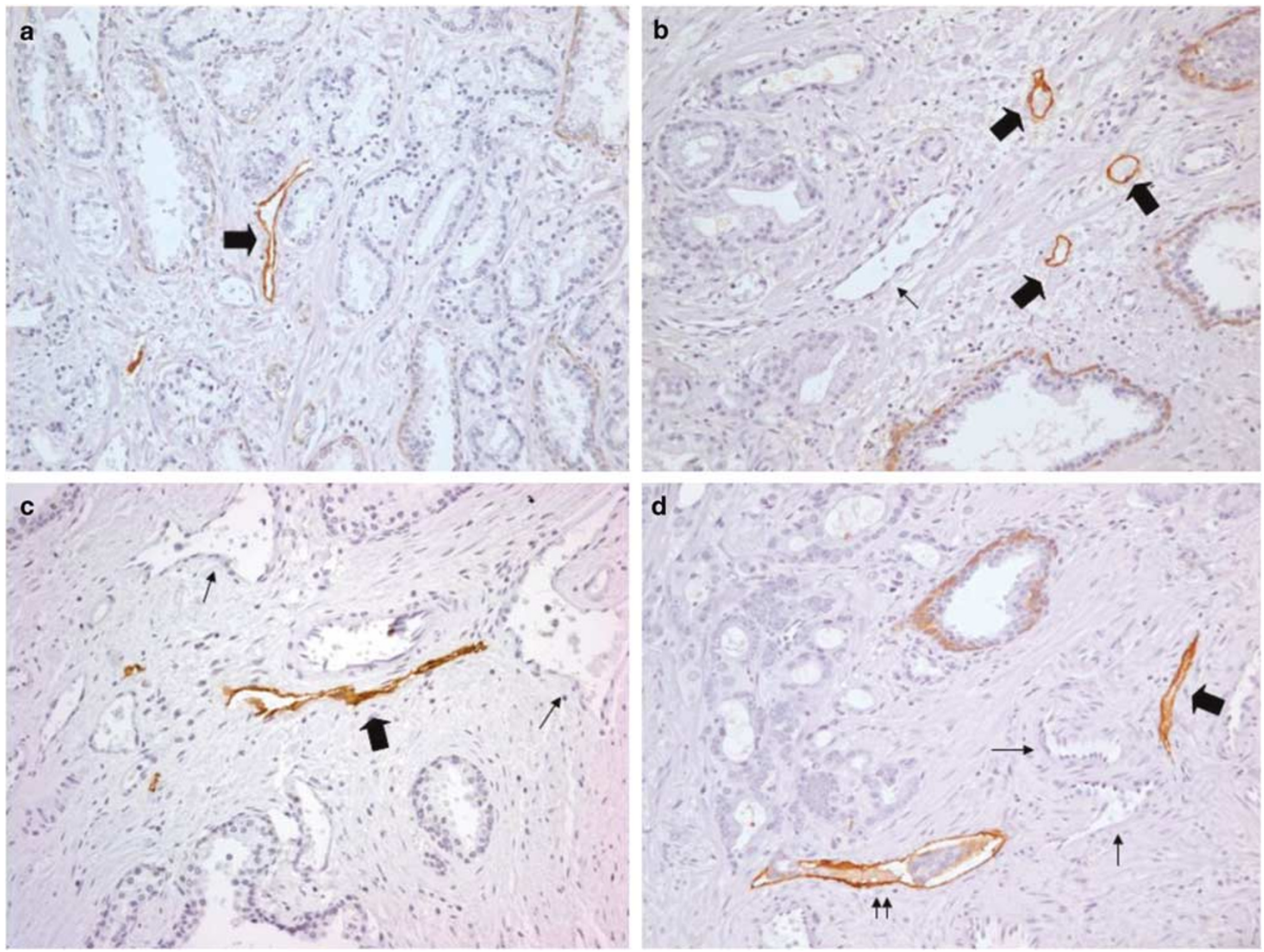

Figure 1 Immunohistochemical detection of lymphatic vessels in prostate glands that harbor prostate adenocarcinoma. Antibody D2-40 was used to detect lymphatic vessels (thick arrows) in intratumoral (a), peritumoral (b) and normal (c) prostate compartments. Note vascular structures (thin arrows) that were negative for D2-40. Lymphatic invasion by prostate cancer cells is shown in (d) (double arrows), with a cluster of cancer cells within the D2-40 positive lymphatic space. The basal cells in benign prostatic glands were also positive for D2-40.

\section{Statistical Analysis}

Paired sample $t$-test was used to compare the lymphatic vessel density of the three prostate compartments. In addition, Fisher's exact test was used to correlate the lymphatic vessel density of the three compartments and lymphatic vessel invasion with regional lymph node metastasis, Gleason grade, extraprostatic extension and other pathological parameters. A $P$-value $<0.05$ was considered statistically significant.

\section{Results}

\section{Lymphatic Vessel Density in Three Different Compartments of Prostate Adenocarcinoma-Bearing Prostate Gland}

Similar to other studies, this study showed that the antibody D2-40 was specific for the lymphatic endothelial cells, and it did not stain the vascular endothelial cells (Figure 1), although this antibody did stain the basal cells of benign prostate glands. The intratumoral, peritumoral and normal prostate lymphatic vessel density were $0.91 \pm 0.80$, $1.54 \pm 0.68$ and $1.58 \pm 0.96 / \mathrm{mm}^{2}$, respectively. The intratumoral lymphatic vessel density was significantly lower than the peritumoral or normal lymphatic vessel density $(P<0.001)$, and the lymphatic vessel density of the latter two compartments was not different $(P=0.81$, Table 2). The lymphatic vessel density of three zones was not significantly different between cases with lymph node metastasis and those without $(P>0.05$, Table 2). The intratumoral and normal prostate lymphatic vessel density did not correlate with any other pathological parameters, including lymph node metastasis, Gleason score, tumor volume, extraprostatic extension, seminal vesicle invasion or surgical margins. The peritumoral lymphatic vessel density, however, correlated with the Gleason score $(P=0.016)$. Prostate adenocarcinoma with higher Gleason scores had higher peritumoral lymphatic vessel density (Table 3). 
Table 2 Lymphatic vessel density in prostate glands with prostate adenocarcinoma

\begin{tabular}{lccc}
\hline Prostate compartments & All cases $(\mathrm{n}=33)$ & + Lymph node metastasis $(\mathrm{n}=14)$ & \\
\hline Lymphatic vessel density $\left(/ \mathrm{mm}^{2}\right)$ Mean \pm s.d. (range) & & $1.08 \pm 0.97(0.18-3.59)$ & $0.56 \pm 0.41(0-1.4)$ \\
Intratumoral & $0.91 \pm 0.80(0-3.59)$ & $1.48 \pm 0.76(0.47-2.79)$ & $1.54 \pm 0.58(0.78-2.66)$ \\
Peritumoral & $1.54 \pm 0.68(0.47-2.79)$ & $1.87 \pm 1.20(0.35-4.78)$ & $1.41 \pm 0.56(0.45-2.40)$ \\
Normal & $1.58 \pm 0.96(0.35-4.78)$ & & \\
\hline
\end{tabular}

For all cases combined, $P<0.001$ for intratumoral vs peritumoral and intratumoral vs normal, and $P=0.81$ for peritumoral vs normal.

$P$-values for the lymphatic vessel density of the intratumoral, peritumoral and normal zones were all $>0.05$ between cases with+lymph node metastasis and those with-lymph node metastasis.

\section{Lymphatic Vessel Invasion in Three Compartments of Prostate Adenocarcinoma-Bearing Prostate Gland}

Lymphatic vessel invasion defined as prostate adenocarcinoma cells with D2-40 positive vascular spaces (Figure 1) was detected in 10 cases, including six cases with lymphatic vessel invasion present only in the intratumoral compartment, two cases only in the peritumoral compartment, and in three cases in both compartments. No lymphatic vessel invasion was identified in the normal prostate compartment. Lymphatic vessel invasion was present in a significantly higher percentage of cases with lymph node metastasis (9/14, 62.3\%), as compared to those without lymph node metastasis (1/12, 8.3\%, $P<0.01$ by $\chi^{2}$ test). The presence of lymphatic vessel invasion (intratumoral and peritumoral compartments combined) correlated with lymph node metastasis $(P=0.005$, Table 3$)$. In addition, lymphatic vessel invasion also correlated with Gleason score, seminal vesicle invasion and positive surgical margins. Interestingly, only peritumoral lymphatic vessel invasion correlated with lymph node metastasis $(P=0.04$, Table 3$)$, while intratumoral lymphatic vessel invasion did not $(P=0.08)$. The positive predictive value of lymphovascular invasion (peritumoral and intratumoral combined) for the lymph node metastasis was $90 \%$ (9/10), while that of peritumoral lymphovascular invasion was $100 \%$ (5/5), and that of intratumoral lymphovascular invasion was $77.8 \%$ (7/9). The sensitivity to predict regional lymph node metastasis was $64.3 \%$ (9/14) for lymphovascular invasion (peritumoral and intratumoral combined), $37.2 \%$ (5/14) for peritumoral lymphovascular invasion, and 50.0\% (7/14) for intratumoral lymphovascular invasion.

\section{Correlation of Other Pathological Parameters}

Lymph node metastasis strongly correlated with extraprostatic extension $(P<0.001)$ and marginally correlated with large tumor volume $(P=0.052)$ and Gleason score $(P=0.07)$. Extraprostatic extension correlated with Gleason score $(P=0.02)$ and tumor volume $(P<0.001)$. Seminal vesicle invasion correlated with lymphatic vessel invasion, both intratumoral $(P=0.027)$ and peritumoral $(P=0.001)$,
Gleason score $(P=0.003)$, lymph node metastasis $(P<0.001)$ and extraprostatic extension $(P=0.012)$. Surgical margin status correlated with lymphatic vessel invasion $(P=0.042)$, tumor volume $(P=0.008)$ and extraprostatic extension $(P=0026)$.

\section{Discussion}

In this study, we have shown that (1) the lymphatic vessel density is reduced in the intratumoral compartment compared to the peritumoral and normal prostate compartments, while the latter two have similar lymphatic vessel density; (2) peritumoral lymphatic invasion correlates better with the regional lymph node metastasis than intratumoral lymphatic vessel invasion; (3) Lymphatic vessel invasion also correlates with other pathological parameters, including Gleason score, seminal vesicle invasion and positive surgical margins.

The existence of lymphatic vessels has been described in benign prostatic tissues. ${ }^{24} \mathrm{Up}$ to date, only one other study has examined the distribution of lymphatic vessels in prostate adenocarcinoma. In prostate adenocarcinoma specimens, Trojan et $a l^{21}$ found that lymphatic vessel density was significantly higher in benign hyperplastic prostate tissue than in nontumorous regions, including normal and peritumoral regions. In addition, the lymphatic vessel density in prostate adenocarcinoma region was significantly decreased compared to that in peritumoral and normal prostate regions. These findings, also confirmed by our current study, suggest that prostate adenocarcinoma may not induce active lymphatic proliferation, or lymphangiogenesis. In contrast, prostate adenocarcinoma actually destroys intratumoral lymphatic vessels. It is also possible that lymphangiogenesis maybe induced by prostate adenocarcinoma in the intratumoral compartment, albeit not as to the same degree as the destruction by prostate adenocarcinoma, and the net result is an overall decrease of lymphatic vessel density in the intratumoral compartment. Additionally, prostate adenocarcinoma cells may secret inhibitors of lymphangiogenesis. It seems surprising at first given that several studies have shown the expression of several lymphangiogenic factors, including $\mathrm{VEGF} \mathrm{C}$ and $\mathrm{D}$, and their receptor 


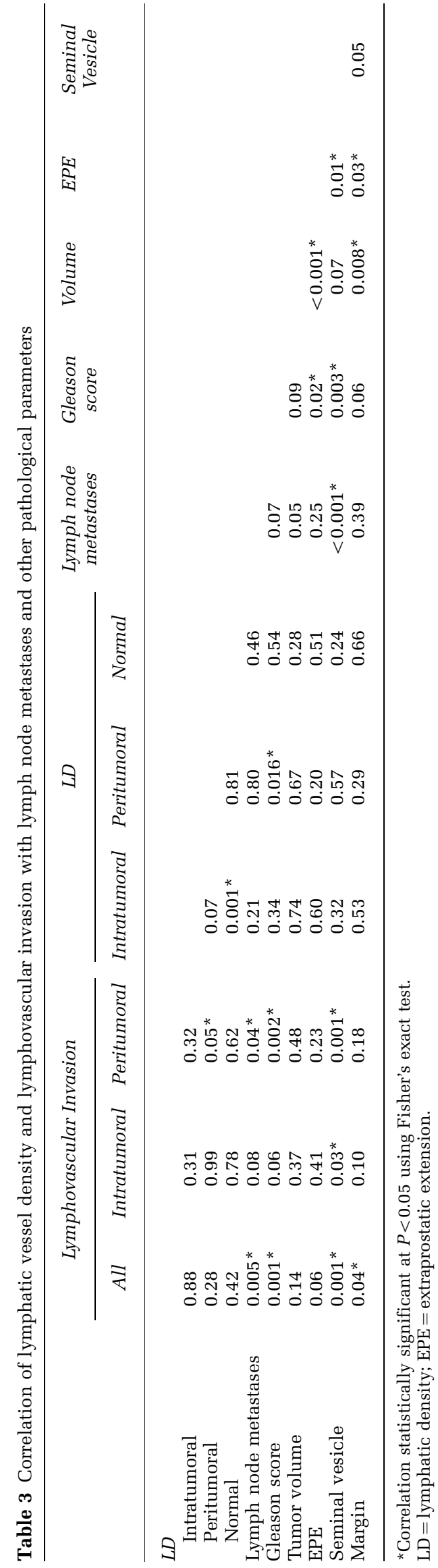

(VEGFR-3) were elevated in prostate adenocarcinoma cells. ${ }^{17-20}$ However, these studies did not correlate the increased expression of these lymphangiogenic factors with increased lymphatic vessel density in prostate adenocarcinoma. Although we did not study the expression of these lymphangiogenic factors in prostate adenocarcinoma, our study, together with the study by Trojan et al, provides strong evidence that active lymphangiogenesis does not play a role in lymphatic invasion and lymph node metastasis. Lack of lymphangiogenesis is not unique to prostate adenocarcinoma, as similar findings were also reported in animal models and other human cancers, ${ }^{25,26}$ and most recently in breast cancer. ${ }^{12,13}$ However, it should be stressed that the lack of lymphangiogenesis in prostate adenocarcinoma and other cancers does not negate the possible role of these lymphangiogenic factors in lymphatic invasion and lymph node metastasis. It is possible that these factors may induce ultrastructural changes in lymphatic vessels that may facilitate the access of prostate adenocarcinoma cells into lymphatic circulation.

Our study suggests that the lymphatic vessels in the peritumoral compartment are important for regional lymph node metastasis. The peritumoral lymphatic vessel density inversely correlates with Gleason grade, and it correlates with the regional lymph node metastasis $(P=0.04)$. On the other hand, intratumoral lymphatic vessel density does not correlate with other pathological parameters, including Gleason grade and tumor volume, nor does it correlate with lymph node metastasis $(P=0.08)$, suggesting that the intratumoral lymphatic vessels may not be as important for tumor growth and invasion into lymphatic vessels as the peritumoral ones. However, one needs to be cautious since the number of cases studied is small and there is no tremendous difference between the peritumoral and intratumoral lymphovascular invasion in their association with regional lymph node metastasis. In addition, lymphovascular invasion involving either peritumoral or intratumoral compartment is strongly associated with regional lymph node metastasis $(P=0.005)$. Both, therefore, should be regarded as related to lymph node metastases and further studies are needed to clarify their perspective roles. However, the sensitivity of lymphovascular invasion to predict lymph node metastasis is low (64.3\%). Our study also showed that lymph node metastasis is strongly associated with seminal vesicle invasion, and marginally associated with large tumor volume and high Gleason score. Therefore, other adverse pathological parameters would also indicate a high probability of lymph node metastasis, even in the absence of lymphovascular invasion.

We also found that lymphatic vessel invasion correlated with seminal vesicle invasion. Most of the seminal vesicle invasion occurs when prostate adenocarcinoma extends out of the prostate gland and then secondarily invades into the base of the 
seminal vesicles where they join the prostate. ${ }^{27}$ Several studies also suggest that prostate adenocarcinoma could involve seminal vesicles via the angiolymphatic route. ${ }^{27,28}$ Although such view is not widely accepted. In our study, seminal vesicle invasion correlated with both extraprostatic extension and lymphatic vessel invasion. Owing to small number of cases, a multivariate analysis was not performed to delineate the true association between seminal vesicle invasion, lymphovascular invasion and extraprostatic extension. Although statistical association does not prove a causal relationship, it nevertheless raises the possibility that both extraprostatic extension and lymphatic vessel invasion may contribute to the seminal vesicle invasion.

Our study potentially has several important implications. First, since the peritumoral lymphatic vessels maybe functionally more important than the intratumoral lymphatic vessels, future studies on lymphangiogenesis in prostate adenocarcinoma should focus in this compartment in addition to the intratumoral compartment. Second, the positive predictive value for lymphovascular invasion (peritumoral and intratumoral combined), especially the peritumoral lymphovascular invasion, to predict lymph node metastasis is high, finding lymphovascular invasion in prostate adenocarcinoma, therefore, implies a high likelihood of lymph node metastasis. In our opinion, the lymphatic vessel invasion should be considered as an important pathological parameter to look for and document when evaluating a radical prostatectomy, especially in cases where lymphadenectomy is not performed, or lymph node metastasis in not found by routine histological examination.

\section{Acknowledgements}

We would like to thank Terry Daly and Angela Thomas of Signet Laboratories, Dedham, MA, USA, for providing D2-40 antibody used in this study.

\section{References}

1 Hanahan D, Weinberg RA. The hallmarks of cancer. Cell 2000;100:57-70.

2 Pepper MS. Lymphangiogenesis and tumor metastasis: myth or reality? Clin Cancer Res 2001;7:462-468.

3 Stacker SA, Achen MG, Jussila L, et al. Lymphangiogenesis and cancer metastasis. Nat Rev Cancer 2002; 2:573-583.

4 Stacker SA, Baldwin ME, Achen MG. The role o tumor lymphangiogenesis in metastatic spread. FASEB J 2002;16:922-934.

5 Baldwin ME, Stacker SA, Achen MG. Molecular controls of lymphangiogenesis. Bio Essays 2002;24: 1030-1040.

6 Nathanson SD. Insights into the mechanisms of lymph node metastasis. Cancer 2003;98:413-423.
7 Schoppmann SF, Bayer G, Aumayr K, et al. Austrian Breast and Colorectal Cancer Study Group. Prognostic value of lymphangiogenesis and lymphovascular invasion in invasive breast cancer. Ann Surg 2004; 240:306-312.

8 Franchi A, Gallo O, Massi D, et al. Tumor lymphangiogenesis in head and neck squamous call carcinoma. A morphometric study with clinical correlations. Cancer 2004;101:973-978.

9 Hashimoto I, Kodama J, Seki N, et al. Vascular endothelial growth factor-C expression and its relationship to pelvic lymph node status in invasive cervical cancer. Br J Cancer 2001;85:93-97.

10 Rubbia-Brandt L, Terris B, Giostra E, et al. Lymphatic vessel density and vascular endothelial growth factor$\mathrm{C}$ expression correlate with malignant behavior in human pancreatic endocrine tumors. Clin Cancer Res 2004;10:6919-6928.

11 Dadras SS, Paul T, Bertoncini J, et al. Tumor lymphangiogenesis: a novel prognostic indicator for cutaneous melanoma metastasis and survival. Am J Pathol 2003;162:1951-1960.

12 Williams CSM, Leek DR, Robson MA, et al. Absence of lymphangiogenesis and intratumoural lymph vessels in human metastatic breast cancer. J Pathol 2003;200:137-274.

13 Vleugel MM, Bos R, van der Groep P, et al. Lack of lymphangiogenesis during breast carcinogenesis. J Clin Pathol 2004;57:746-751.

14 McDavid K, Lee J, Fulton JP, et al. Prostate cancer incidence and mortality rates and trends in the United States and Canada. Public Health Rep 2004;119: 174-186.

15 Bono AV. The global state of prostate cancer: epidemiology and screening in the second millennium. BJU Int 2004;94(Suppl 3):1-2.

16 Walsh P. Campbell's Urology, 8th edn. Saunders: London, 2002.

17 Tsurusaki T, Kanda S, Sakai H, et al. Vascular endothelial growth factor-C expression in human prostatic carcinoma and its relationship to lymph node metastasis. Br J Cancer 1999;80:309-313.

18 Zeng Y, Opeskin K, Baldwin ME, et al. Expression of vascular endothelial growth factor receptor-3 by lymphatic endothelial cells is associated with lymph node metastasis in prostate cancer. Clin Cancer Res 2004;10:5137-5144.

$19 \mathrm{Li} \mathrm{R}$, Younes M, Wheeler TM, et al. Expression of vascular endothelial growth factor receptor-3 (VEGFR-3) in human prostate. Prostate 2004;58: 193-199.

20 Jennbacken K, Vallbo C, Wang W, et al. Expression of vascular endothelial growth factor C (VEGF-C) and VEGF receptor-3 in human prostate cancer is associated with regional lymph node metastasis. Prostate 2005;65:110-116.

21 Trojan L, Michel MS, Rensch F, et al. Lymph and blood vessel architecture in benign and malignant prostatic tissue: Lack of lymphangiogenesis in prostate carcinoma assessed with novel lymphatic marker lymphatic vessel endothelial hyaluronan receptor (LYVE-1). J Urol 2004;172:103-107.

22 Kahn HJ, Marks A. A new monoclonal antibody, D2-40, for detection of lymphatic invasion in primary tumors. Lab Invest 2002;82:1255-1257.

23 Fogt F, Zimmerman RL, Ross HM, et al. Identification of lymphatic vessels in malignant, adenomatous and 
normal colonic mucosa using the novel immunostain D2-40. Oncol Rep 2004;11:47-50.

24 Furusato M, Mostofi FK. Intraprostatic lymphatics in man: light and ultrastructural observations. Prostate 1980;1:15-23.

25 Leu AJ, Berk DA, Lymboussaki A, et al. Absence of functional lymphatics within a murine sarcoma: a molecular and functional evaluation. Cancer Res 2000;60:4324-4327.

26 Padera TP, Kadambi A, di Tomaso E, et al. Lymphatic metastasis in the absence of functional intratumor lymphatics. Science 2002;296:1883-1886.
27 Ohori M, Scardino PT, Lapin SL, et al. The mechanisms and prognostic significance of seminal vesicle involvement by prostate cancer. Am J Surg Pathol 1993;17:1252-1261.

28 Graham Jr SD, Napalkov P, Watts L, et al. Microvascular invasion of the seminal vesicles in adenocarcinoma of the prostate. Prostate 1996;28: 359-363.

29 Renshaw AA, Chang H, D'Amico AV. Estimation of tumor volume in radical prostatectomy specimens in routine clinical practice. Am J Clin Pathol 1997; 107:704-708. 